\title{
Portable Machine to Machine Systems for Distance Phisico-Chemical Parameters of River Water Monitoring
}

\author{
Heru Supriyono $^{1}$, Naufan Faldiansyah ${ }^{1}$, Kun Harismah ${ }^{2}$ \\ ${ }^{1}$ Department of Electrical Engineering, Universitas Muhammadiyah Surakarta, Indonesia, \\ Heru.Supriyono@ums.ac.id, D400160085@ums.ac.id \\ ${ }^{2}$ Department of Chemical Engineering, Universitas Muhammadiyah Surakarta, Indonesia,
} Kun.Harismah@ums.ac.id

\begin{abstract}
River water quality monitoring is very important since river water is also used as a source of fresh water besides for agricultural and farming purposes. Manual river water quality monitoring which is done by taking river water samples in a period of time and analyzing it in the laboratory has limitations in that the actual condition of river water could not be monitored continuously. The objective of this article is to monitor the physico-chemical parameters of river water including potential of Hydrogen $(\mathrm{pH})$, temperature, and Total Dissolved Solid (TDS) continuously in a distance. As a prototype, the proposed architecture facilitates the river water quality could be monitored from a long distance far away from the river location as from the government office building. The proposed system consists of data acquisition part, base station part, wireless data communication system, and cloud for storing and displaying measured parameters. The proposed system was implemented by using two LoRa32u4 boards, a NodeMCU board, and blynk cloud platform. Compared to the commercial measuring instrument, the laboratory scale testing results showed that the measurements of the proposed system have the average measurement difference $(\Delta p)$ of $0.2 \%, 0.7 \%$, and $4.4 \%$ for TDS, $\mathrm{pH}$ and temperature respectively. The proposed system was tested in an actual field where the data acquisition part was located at the river in Ngawi regency while the base station part was placed $100 \mathrm{~m}$ apart. The actual field test results showed that the proposed system was able to measure all three water parameters consistently.
\end{abstract}

Key words: Distance monitoring, machine to machine, river water monitoring, water quality monitoring.

\section{INTRODUCTION}

Water quality of the river is very important to be monitored because besides for agricultural and farm purposes rivers water are also used as sources of drinking water [1]. There are parameters for measuring the river water quality. Among of them, the two physico-chemical parameters commonly monitored are Power of Hydrogen or Potential of Hydrogen $(\mathrm{pH})$ and turbidity or Total Dissolved Substances (TDS) [2]. The level of $\mathrm{pH}$ of water is very important to monitor because it affects the health of fish in aquaculture [3]. Besides $\mathrm{pH}$ and TDS, there is a physical water parameter which is also usually measured, i.e. temperature [4] since water temperature would affect other biological or chemical water parameters [5].

Chemical pollutant substances such as from domestic, industrial, agricultural and farming could be able to raise water temperature above its normal condition in accordance with the environment. The common method for river water quality monitoring is by taking a water sample of river water and then conducting laboratory analysis such as conducted in [6]. This method is not facilitating continuous and real time monitoring since the condition of river water could be changed continuously according to time.

Distance river water monitoring means that the persons who monitor the river water quality are located at the river but in some distance from the river. Attempts for distance monitoring water quality has been proposed by researchers. There were various technologies utilized by researchers such as the combination of using $433 \mathrm{MHz}$ data communication and Zigbee protocol [7]. This architecture has limitations since the data from the station board was sent to the Personal Computer (PC) offers less mobility. Furthermore, Zigbee protocol was proposed for water quality monitoring using wireless sensor networks (WSN) [8]. The limitation of Zigbee protocol is its data communication range so that it is considerably not suitable if implemented for large or long spans of area such as in a river [9]. Another distance monitoring system of water quality was proposed by researchers in [10] where wireless fidelity (wifi) was used to connect the data acquisition unit to the display instrument such as smartphone or PC. The use of wifi of this system made the measured data were only could be accessed if wifi networks were available so that the deployment of such systems in the actual field or remote area such as the river would be a concern. 
Website technology enables the measured data of water monitoring systems to be accessed anywhere by using internet access such as proposed in [11] however the dedicated web server has to be located around the data acquisition unit so that it is less practical to be implemented in the actual field of river water monitoring system. Another technology used by researchers for facilitating distance water quality monitoring is cloud computing technology where the measured data could be stored and visualized in it for example ThingSpeak platform [12], and Ubidots platform [13]. By using cloud technology, the water quality parameters could be displayed on a smartphone or computer.

The objective of this article is to monitor river water quality monitoring continuously in a distance. The development steps would be outlined first before the testing process both for laboratory scale test and actual field testing. Finally the discussion including the comparison of the proposed system to other existing systems would be presented.

\section{MATERIALS AND METHOD}

\subsection{System Architecture}

The proposed system for monitoring river water pollution has machine to machine architecture. The proposed system has various units involving a data acquisition unit for measuring water parameters, data communication for sending the water parameters data, storage or database or cloud for storing water parameters and a final unit for displaying the water either in the form of numeric or graphics such as a smartphone or computer. By using machine to machine architecture means that the processes from the data acquisition to data displaying were done automatically without human intervention. There are three water parameters monitored involving $\mathrm{pH}$, temperature, and TDS. These three parameters are commonly used in the water quality monitoring [5].

Physically, the proposed monitoring system was implemented in two parts namely transmitter and receiver parts as can be seen in Figure 1. The transmitter part consists of three sensors for sensing water parameters namely TDS sensor SKU SEN0244, pH sensor SKU SEN0161, and temperature sensor DS18B20. All the three sensors are connected to LoRa32u4 board in which the data produced by sensors would be processed and sent to the receiver part using $433 \mathrm{MHz}$ wireless data communication. The sent data is then received by the LoRa32u4 board. The data are then displayed by using Liquid Crystal Display (LCD) and sent to NodeMCU ESP8266 microcontroller. The data are then sent to the IoT server cloud by NodeMCU ESP8266 so that it could be accessed by using the Blynk application of the smartphone. The proposed system architecture is different from the system proposed in [14] where the data acquisition unit was directly connected to the cloud without a base station.

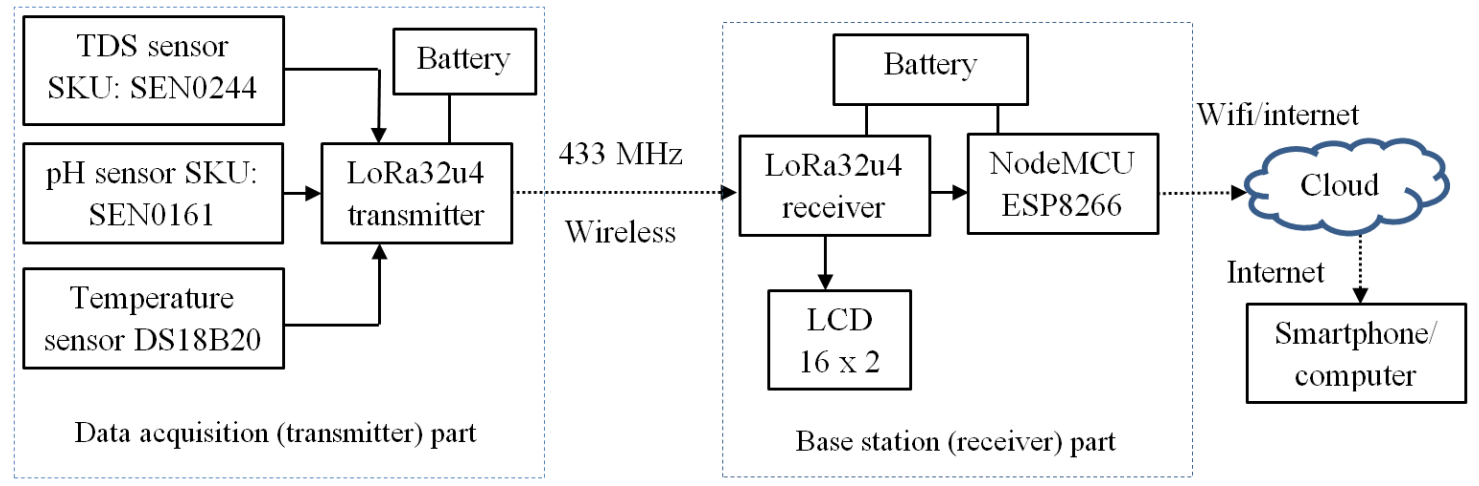

Figure 1: Block Diagram of the Proposed Distance Monitoring Systems

LoRa $32 \mathrm{u} 4$ was chosen as the main processor because this board contained an ATMega32u4 microcontroller and LoRa Ra-02 a $433 \mathrm{MHz}$ wireless data communication module. Based on the specification, LoRa Ra-02 could be used for transmitting data in the range of up to $20 \mathrm{~km}$ if it is equipped with an antenna. Furthermore, implementation of LoRa board for long distance monitoring systems has advantages such as it needs lower cost for deployment and maintenance compared to other technology such as wireless sensor networks (WSN) technology [15]. In addition, the use of WSN for long distance river water monitoring such as proposed in [16] will need more data communication instruments involving gateway in order to send the data in longer distance than standard WSN protocol for example Zigbee or Wifi.

LoRa technology was also reported to be applied for transmitting some building-related parameters monitoring such as temperature, humidity, light, and presence in real time [17]. The $433 \mathrm{MHz}$ wireless data communication has also been used by research in portable systems for detecting flammable gas and it provided satisfactory results for moving systems [18]. TDS sensor SKU SENO244 is able to sense solid substances dissolved in the water with accuration in the range of $\pm 10 \%$ in the working temperature of $25^{\circ} \mathrm{C}$. SKU SEN0161 $\mathrm{pH}$ sensor has characteristics as able to sense $\mathrm{pH}$ level of water 
or solution between 0 and 14 with accuration level in the range of \pm 0.1 scale in the working temperature of $25^{\circ} \mathrm{C}$ with response time $\leq 1$ minute. The third sensor used in the proposed system, temperature sensor DS18B20 has characteristics of waterproof so that it could be used for measuring temperature of water or solution in the range of between $-55^{\circ} \mathrm{C}$ and $125^{\circ} \mathrm{C}$ with accuration level of $0.5^{\circ} \mathrm{C}$.

\subsection{Hardware Circuit Implemetation}

The system architecture presented in Figure 1 was implemented in the hardware circuit in Figure 2 and 3 for transmitter and receiver parts respectively.

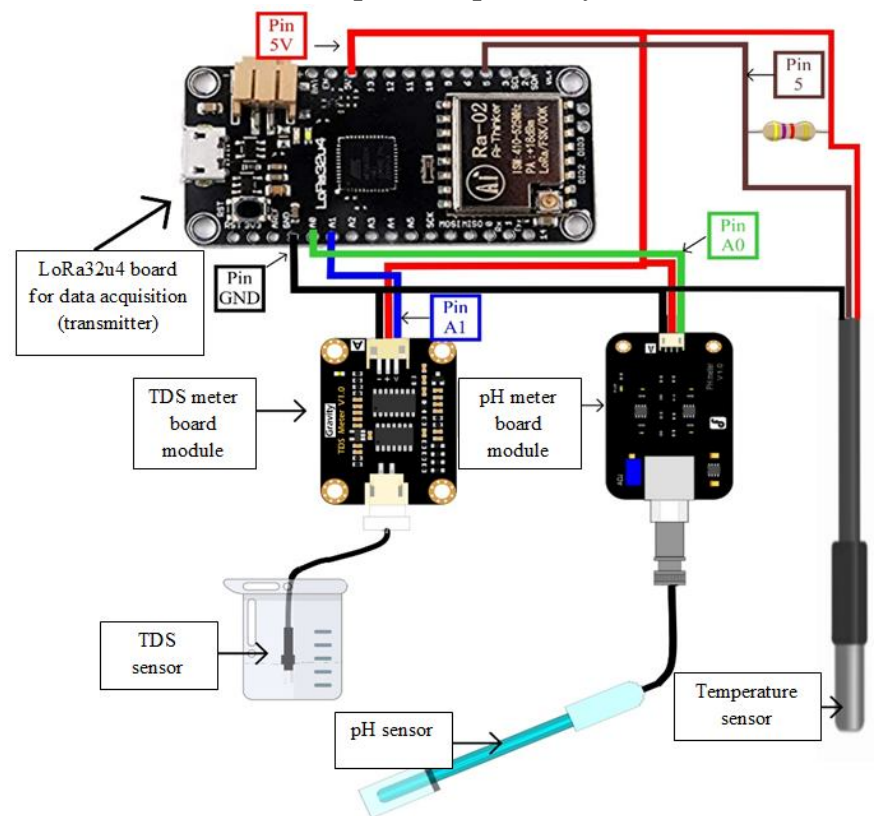

Figure 2: Hardware Circuit of the Transmitter Part

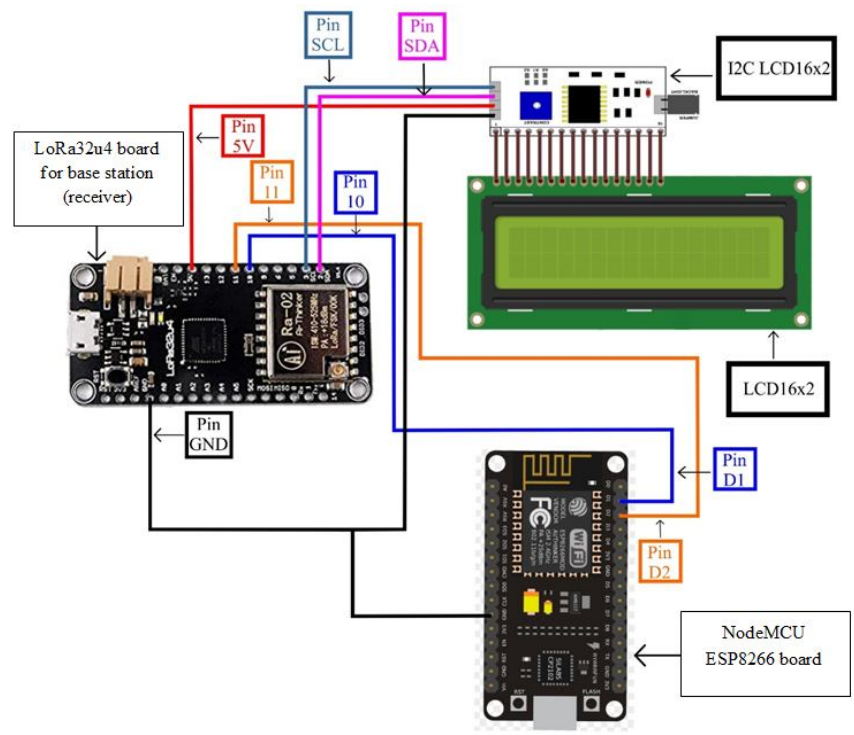

Figure 3: Hardware Circuit of the Receiver Part

All three microcontrollers, sensor modules, and sensors used in the proposed systems need a 5 Direct Current Volt (DCV) power source. The power source used was rechargeable Li-ion batteries with voltage of 3.7 DCV which then was increased to 5 DCV by using a step up transformer to meet the requirement. For the data acquisition (transmitter) part, the output of sensors were connected to the LoRa32u4 board (transmitter) as follows. The output of the temperature sensor was connected to Pin 5 of the LoRa32u4 board. The TDS sensor was connected to the TDS meter board module and its output was then connected to Pin A1 of LoRa32u4 board. Similarly, the $\mathrm{pH}$ sensor was connected to the $\mathrm{pH}$ meter board module and its output was then connected to pin A0 of LoRa32u4 board. In the base station (receiver) part, pin 10 and 11 of LoRa32u4 board were connected to pin D1 and D2 of NodeMCU respectively. Furthermore, Pin SDA and SCL of LoRa32u4 were connected to I2C boards of LCD 16x2.

\subsection{Software Design}

The computation process in the proposed system could be seen in the flowchart of Figure 4.

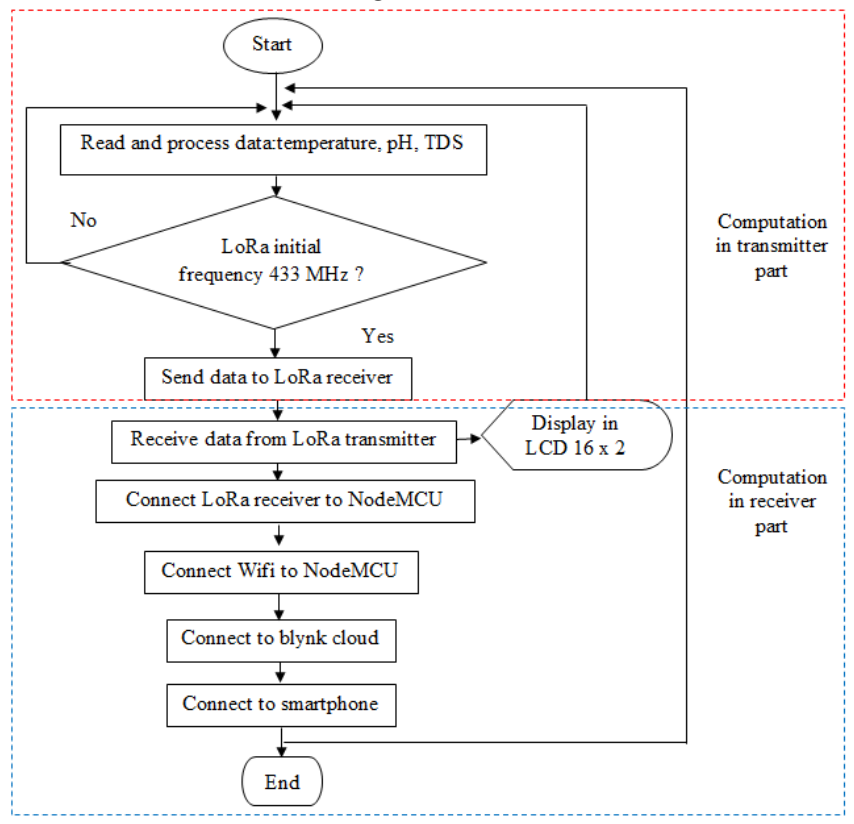

Figure 4: Flowchart Showing General Computation of Transmitter and Receiver Part

There are two computation processes namely computation process in the transmitter part and computation process in the receiver part. In the transmitter part, the computation process is performed by the LoRa $32 \mathrm{u} 4$ board for the transmitter while in the receiver part the computation process is performed by the LoRa $32 \mathrm{u} 4$ board for receiver and the NodeMCU board. The first step of a whole computation process is LoRa $32 \mathrm{u} 4$ for which the transmitter will read the data produced by three sensors: temperature, $\mathrm{pH}$, TDS. After that the LoRa will compute these data to produce the final value. Furthermore, LoRa will check whether the data communication is ready in its operating frequency, i.e. $433 \mathrm{MHz}$ if yes so it will send all the data to the receiver part otherwise it will reset and start the 
initial step, i.e. read the data output of the three sensors. At the receiver part, the computation process is started by the LoRa board receiving the measurement data from the transmitter part. These data are then displayed in LCD 16x2. Moreover, LoRa for the receiver will be connected to the NodeMCU so that the data could be accessed via wifi or via blynk framework. The softwares for LoRa and NodeMCU boards was developed by using Arduino Integrated Development Measurement (IDE).

\subsection{Testing Plan}

The proposed monitoring system was tested in the laboratory and the actual field condition. In the laboratory scale testing, the proposed system would be used to measure the three variables of water and the results would be compared to those of by commercial measuring instrument. This kind of testing is useful to understand how close the measurement results are by using the proposed system to commercial measuring instruments. The final test was field testing where the proposed system was tested in the actual river condition.

\section{RESULTS AND ANALYSIS}

\subsection{Obtained Systems}

All hardware circuits of the proposed system were placed in the acrylic box to protect from water splash and dent. There are two acrylic boxes namely box for transmitter part which has dimension of $22 \mathrm{~cm}$ (length) $\times 21 \mathrm{~cm}$ (width) $\times 8 \mathrm{~cm}$ (height) while box for receiver has dimension of $29 \mathrm{~cm}$ (length) $\times 16 \mathrm{~cm}$ (width) $\times 8 \mathrm{~cm}$ (height). The sketch of the two boxes can be seen in Figure 5. There are holes and windows of two boxes for placing wires, instruments and as a part of cooling systems.
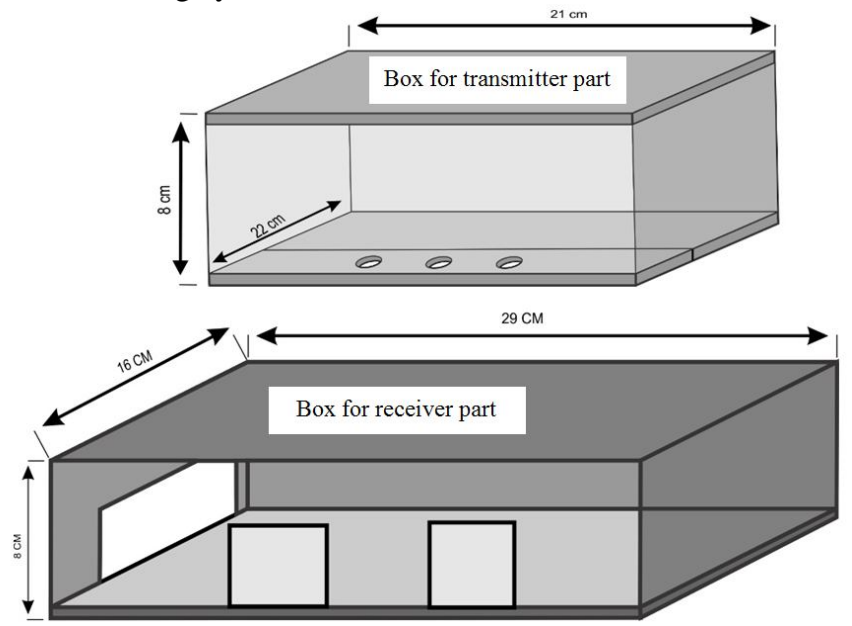

Figure 5: Acrylic Boxes for Housing Hardware Circuit of Transmitter Part and Receiver Part

In the receiver part, all three measured data of the proposed system were displayed at the same time in the LCD as can be seen in Figure 6 where the first row stated the variable, i.e. temperature, TDS and $\mathrm{pH}$ while the second row stated its corresponding value. Besides, the data also could be displayed on smartphones by using a Blynk application because all measurement data have been sent to the Blynk cloud server by the NodeMCU board. However, the display using blynk application is very dependent on the availability of internet access. If needed, the data also can be displayed on computer or laptop, however, the computer or laptop has to install the Android emulator first to be able to access the blynk application. The display of measurement data on the smartphone and laptop can be seen in Figure 7.

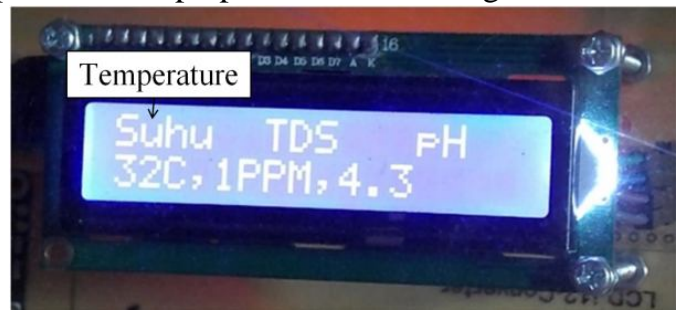

Figure 6: LCD Displaying All Three Measured Variables at the Same Time

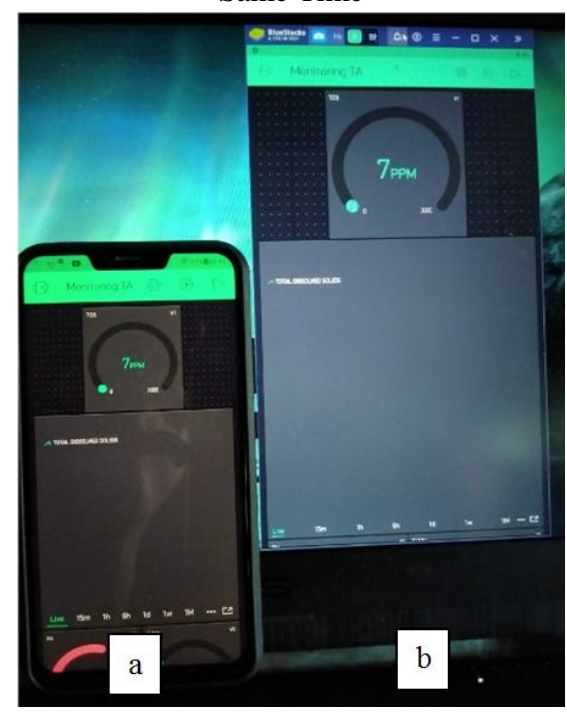

Figure 7: Display of Measured Variables at: a) Smartphone, b) Computer/Laptop

There are three gauges with three graphics displayed in the Blynk application for three variables as can be seen in Figure 8 . The gauge displays the variable in numeric and animated scale while the graphic displayed the measured value continuously. All gauges and graphics are refreshed every one second to display the current measurement value. The data from the Blynk application could be emailed in the form of *.csv file format. 

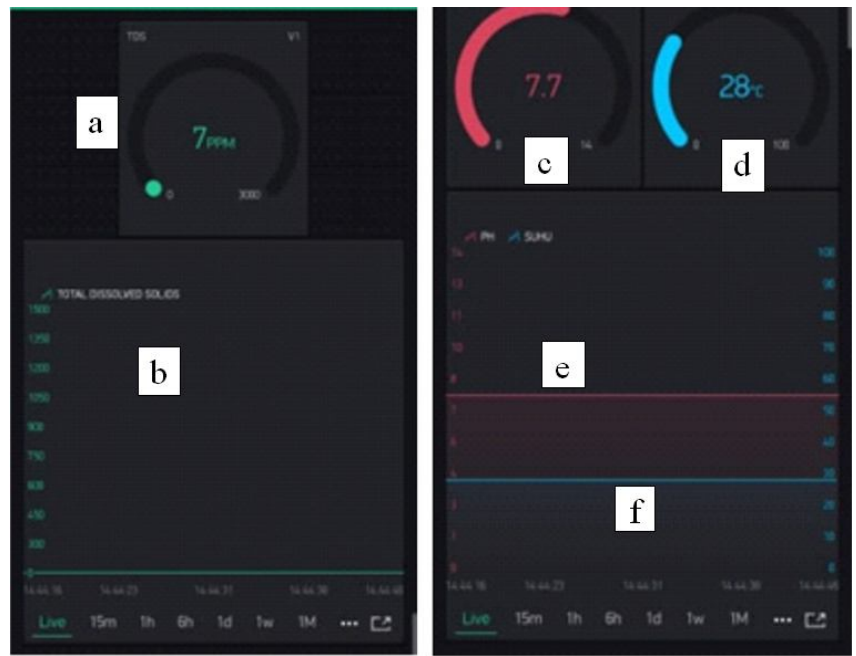

Figure 8: Display of the Blynk Application at the Smartphone: a) Gauge for TDS, b) Graphic for TDS, c) Gauge for $\mathrm{pH}$, d) Gauge for

Temperature, e) Graphic for $\mathrm{pH}, \mathrm{f}$ ) Graphic for Temperature

\subsection{Laboratory Scale Testing Results}

Laboratory scale test was conducted for every variable one by one. The test protocol could be explained as follows. First, the water sample was prepared. Second, the measurement by using the proposed system and commercial measuring instrument was conducted on the prepared water sample. Third, as also used by previous researchers such in [19], the measurement results were collected and compared to produce the measurement difference value calculated using Equation (1) and (2).

$$
\begin{gathered}
\Delta=|c-a| \\
\Delta p=\frac{\Delta}{c} \times 100 \%
\end{gathered}
$$

Where $\Delta$ is the measurement difference value, $c$ is the measurement result by using a commercial measuring instrument, $a$ is the measurement result by using the proposed system, and $\Delta p$ is the measurement difference in percent. Figure 9 shows the photograph documentation of the laboratory scale testing process for all three variables.

The first test was the TDS test. The test was conducted on two water samples: (i) fresh water contained with soil and ash and (ii) laundry waste water. These two water samples were chosen to model two kinds of pollution sources of the river, i.e. pollution from farming activities and domestic waste. For each sample, measurement was conducted continuously in ten minutes and the data were recorded every two minutes so that there were five data samples. The measurement results of the proposed system were compared to those of a commercial TDS meter. The testing results can be seen in Table 1. The table showed that the measurement results of the proposed system were inline with those of by a commercial measuring instrument with average $\Delta$ was 1.3 ppm or average $\Delta p$ was equal to $0.2 \%$. These results are very encouraging that the proposed system is feasible to use.

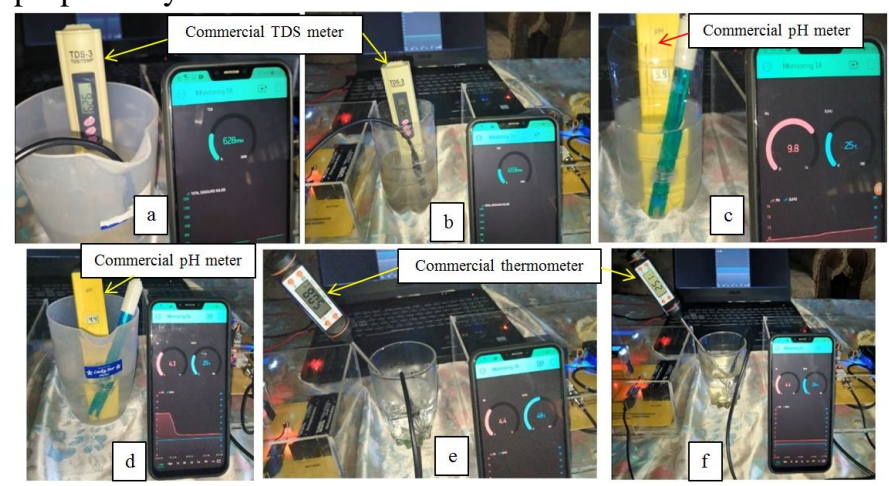

Figure 9: Process of Measurement Testing and its Comparison to Commercial Measuring Instrument: TDS Measurement Test (a and b), $\mathrm{pH}$ Measurement Test (c and d), and Temperature Measurement Test (e and $\mathrm{f}$ )

\begin{tabular}{|c|c|c|c|c|}
\hline Samples & $\begin{array}{c}a \\
(\mathbf{p p m})\end{array}$ & $\begin{array}{c}c \\
(\mathbf{p p m})\end{array}$ & $\begin{array}{c}\Delta \\
(\mathbf{p p m})\end{array}$ & $\Delta p_{(\%)}$ \\
\hline \multirow{5}{*}{$\begin{array}{l}\text { Fresh water } \\
\text { contained with } \\
\text { soil and ash }\end{array}$} & 457 & 458 & 1 & 0.2 \\
\hline & 459 & 458 & 1 & 0.2 \\
\hline & 459 & 458 & 1 & 0.2 \\
\hline & 459 & 458 & 1 & 0.2 \\
\hline & 459 & 458 & 1 & 0.2 \\
\hline \multirow{5}{*}{$\begin{array}{c}\text { Laundry waste } \\
\text { water }\end{array}$} & 628 & 626 & 2 & 0.3 \\
\hline & 628 & 626 & 2 & 0.3 \\
\hline & 625 & 626 & 1 & 0.2 \\
\hline & 628 & 626 & 2 & 0.3 \\
\hline & 625 & 626 & 1 & 0.2 \\
\hline \multicolumn{3}{|c|}{ Min } & 1 & 0.2 \\
\hline \multicolumn{3}{|c|}{ Max } & 2 & 0.3 \\
\hline \multicolumn{3}{|c|}{ Average } & 1.3 & 0.2 \\
\hline
\end{tabular}

Table 1: TDS measurement test results

The second laboratory scale testing was $\mathrm{pH}$ measurement testing. The testing was conducted on two samples: (i) water contained with detergent which was base solution and (ii) water contained with acid substances which was acid solution. These two samples were chosen for modelling the pollution from both domestic and industrial wastes. For every sample, the measurements using the proposed system and ATC commercial $\mathrm{pH}$ meter were conducted in ten minutes and the data were collected every two minutes so that there are five measurement data. The test results presented in Table 2 shows that the measurement results by using the proposed systems are very close to those of by a commercial measuring instrument with average $\Delta$ value is equal to 0.05 scale or average $\Delta p$ is equal to $0.7 \%$. 
Table 2: Measurement of $\mathrm{pH}$ testing results and its comparison to commercial $\mathrm{pH}$ meter

\begin{tabular}{|c|c|c|c|c|}
\hline Samples & $a$ & $c$ & $\Delta$ & $\Delta p_{(\%)}$ \\
\hline \multirow{5}{*}{$\begin{array}{c}\text { Water } \\
\text { contained } \\
\text { with } \\
\text { detergent }\end{array}$} & 9.8 & 9.9 & 0.1 & 1.0 \\
\hline & 9.9 & 9.9 & 0 & 0.0 \\
\hline & 9.9 & 9.9 & 0 & 0.0 \\
\hline & 9.9 & 10 & 0.1 & 1.0 \\
\hline & 9.9 & 9.9 & 0 & 0.0 \\
\hline \multirow{5}{*}{$\begin{array}{c}\text { Water } \\
\text { contained } \\
\text { with acid } \\
\text { substances }\end{array}$} & 4.3 & 4.4 & 0.1 & 2.3 \\
\hline & 4.3 & 4.3 & 0 & 0.0 \\
\hline & 4.3 & 4.3 & 0 & 0.0 \\
\hline & 4.2 & 4.3 & 0.1 & 2.3 \\
\hline & 4.2 & 4.3 & 0.1 & 2.3 \\
\hline \multicolumn{3}{|c|}{ Min } & 0 & 0.0 \\
\hline \multicolumn{3}{|c|}{ Max } & 0.1 & 2.3 \\
\hline \multicolumn{3}{|c|}{ Average } & 0.05 & 0.7 \\
\hline
\end{tabular}

The last laboratory scale test was temperature testing. Testing was conducted on hot and cold water samples. The same as for TDS and $\mathrm{pH}$ testing, the temperature testing was conducted in ten minutes with measurement data were conducted every two minutes so that there were five measurement data. It can be noted from the testing results presented in Table 3 that the proposed system has average difference measurement values $(\Delta)$ equal to $1.7^{\circ} \mathrm{C}$ in average or average of $\Delta p$ equal to 4.4 $\%$.

Table 3: Temperature measurement testing results and its comparison to commercial thermometer

\begin{tabular}{|c|c|c|c|c|}
\hline Samples & $\begin{array}{c}a \\
\left({ }^{\circ} \mathbf{C}\right)\end{array}$ & $\begin{array}{c}c \\
\left({ }^{\circ} \mathbf{C}\right)\end{array}$ & $\begin{array}{c}\Delta \\
\left({ }^{\circ} \mathbf{C}\right)\end{array}$ & $\begin{array}{l}\Delta p \\
(\%)\end{array}$ \\
\hline \multirow{5}{*}{$\begin{array}{l}\text { Hot } \\
\text { water }\end{array}$} & 48 & 50 & 2 & 4.0 \\
\hline & 48 & 51 & 3 & 5.9 \\
\hline & 47 & 50 & 3 & 6.0 \\
\hline & 47 & 49 & 2 & 4.1 \\
\hline & 47 & 49 & 2 & 4.1 \\
\hline \multirow{5}{*}{$\begin{array}{l}\text { Cold } \\
\text { water }\end{array}$} & 24 & 25 & 1 & 4.0 \\
\hline & 24 & 25 & 1 & 4.0 \\
\hline & 24 & 25 & 1 & 4.0 \\
\hline & 24 & 25 & 1 & 4.0 \\
\hline & 24 & 25 & 1 & 4.0 \\
\hline \multicolumn{3}{|c|}{ Minimum } & 1 & 4.0 \\
\hline \multicolumn{3}{|c|}{ Maximum } & 3 & 6.0 \\
\hline \multicolumn{3}{|c|}{ Average } & 1.7 & 4.4 \\
\hline
\end{tabular}

\subsection{Field Testing Results}

The field testing was conducted in the river of Sambiroto Village, Padas District, Ngawi Regency. The river is the outtake of the local dam. The visual condition of the river water was slightly brown, not clear, which indicated that there was pollution. The probable pollution sources are involving domestic and farming wastes. The transmitter part of the proposed systems was placed in the river while the receiver part was placed at the house. The distance between transmitter part and receiver part was estimated to be 100 metres. The exact location of the transmitter and receiver parts could be seen in the map of Figure 10. In the test, the transmitter part was installed in the river bank, as can be seen in Figure 11, so that the three sensors could be exposed by the river water.

The field testing was conducted on a sunny day for the testing period of 15 minutes with measurement data for all three variables were manually recorded every minute so that there were 15 data. The test results presented in Table 4 showed that the measurement of all three variables are consistent without abrupt changes because the river condition was visually relatively unchanged in the testing period except for TDS measurement in 6-th minute when a nearby cow farm drained out its waste. It can be noted from the experimentation that in the range of 100 metres LoRa board of transmitter successfully sent the data to the receiver part with relatively good connection. There were some very-short-time data communication lost incidents in the test, around one second, up to five times data loss in one minute. The data lost mainly happened when there was a big vehicle passing by which caused a strong vibration of the street or very noisy vehicle pass though the place where the transmitter part was located because the location of experimentation was very near to the highway.

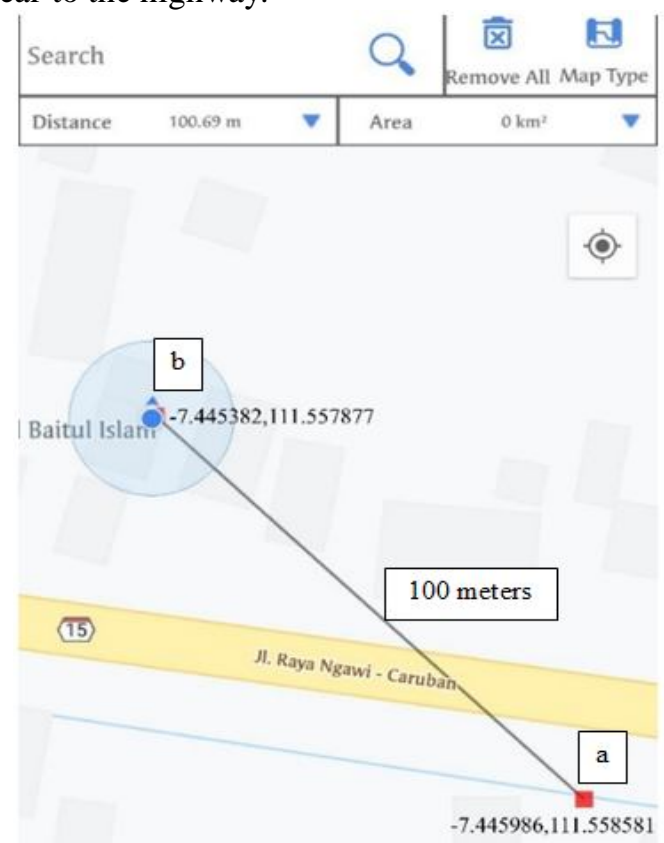

Warung makan 88 ศิ

Figure 10: Maps with Coordinate Showing Location of Field Testing of the Proposed System: a) the Location Where the Transmitter Part including Sensors was Placed for Testing and b) the Location Where the Receiver Part was Placed to Receive Data 


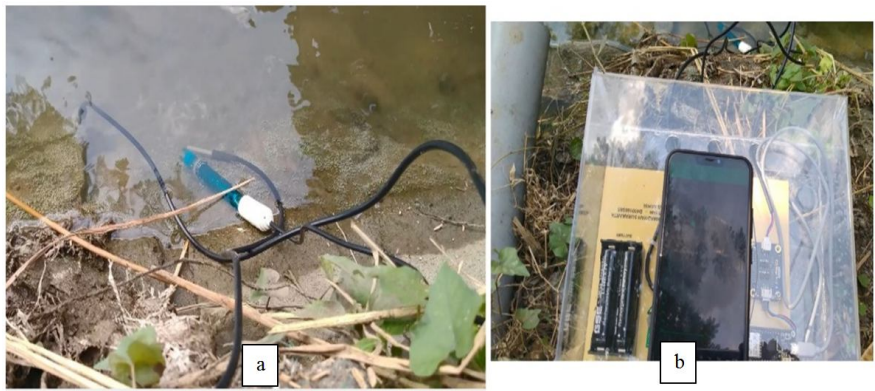

Figure 11: Transmitter Installation at the River Bank During Field Testing: a) the Three Sensors are Expossed to the River Water, and b) the Transmitter Box with Smartphone Just to Show the Exact Coordinate in the Map

Table 4: Testing results of monitoring systems in the river

\begin{tabular}{|c|c|c|c|}
\hline $\begin{array}{c}\text { Time: } \\
\text { i-th minute }\end{array}$ & $\begin{array}{c}\text { TDS } \\
(\mathbf{p p m})\end{array}$ & $\mathbf{p H}$ & $\begin{array}{c}\text { Temperature } \\
\left({ }^{\circ} \mathbf{C}\right)\end{array}$ \\
\hline 1 & 118 & 7.9 & 29 \\
\hline 2 & 118 & 7.9 & 29 \\
\hline 3 & 118 & 7.9 & 29 \\
\hline 4 & 118 & 7.9 & 29 \\
\hline 5 & 118 & 7.9 & 29 \\
\hline 6 & 256 & 7.9 & 29 \\
\hline 7 & 118 & 7.9 & 29 \\
\hline 8 & 118 & 7.9 & 29 \\
\hline 9 & 117 & 7.9 & 29 \\
\hline 10 & 116 & 7.9 & 29 \\
\hline 11 & 116 & 7.9 & 29 \\
\hline 12 & 117 & 7.9 & 29 \\
\hline 13 & 118 & 7.9 & 29 \\
\hline 14 & 117 & 7.9 & 29 \\
\hline 15 & 118 & 7.9 & 29 \\
\hline
\end{tabular}

\subsection{Discussion}

The comparison of proposed systems to similar systems published by other researchers on some aspects is summarised as follows. Compared to other published research results in [7], [8], [10], and [12], the proposed system has shown work as expected when implemented in the actual field since it has been tested on actual river with data acquisition and base station were separated in the distance of $100 \mathrm{~m}$ while most other similar systems were tested in laboratory scale. The use of the blynk cloud application for storing and visualizing the measured data enabled the data could be accessed anywhere so that it supported mobility compared to when the measured data were stored in a dedicated computer. Furthermore, since the data acquisition and base station unit of the proposed system were separated, it offer more practicability for actual river field to setting up the cloud because the base station could be located in the office where the internet connection needed for sending the measured data to cloud would be easily provided compared to other systems which connect directly the data acquisition directly to cloud.
However, the proposed system has weaknesses such as the construction of the data acquisition unit was not floating and well protected against possible environmental conditions including heavy dust and rainfall. Another weakness of the proposed system is the power source. In the development and experimentation process the rechargeable batteries were used. This scenario is less practicable if the proposed system would be implemented in a remote area in a long period of time since it will need frequent activities to check the condition of batteries and possible replacement with the new one. Energy harvesting for example such as proposed in [20] or by using photovoltaic (PV) such as proposed in [8] could be considered to be applied. Moreover, the warning message when any water parameter above its standard such as using buzzer [21], Short Message Services (SMS) of Global System Mobile (GSM) networks [13] or Telegram application [19] would be useful.

\section{CONCLUSION}

Based on the design and test results it could be noted that the proposed monitoring system was able to detect and compute the TDS, pH, and temperature levels of the water. The resulting measurements were then displayed in the LCD $16 \mathrm{x}$ 2 , and Blynk application of smartphone and computer. The laboratory scale testing results showed that the proposed system has consistent measurement results in comparison to that of commercial measuring instruments with average measurement difference ( $\Delta p$ ) was $0.2 \%, 0.7 \%$, and $4.4 \%$ for TDS measurement test, $\mathrm{pH}$ measurement test, and temperature measurement test respectively. The field testing results showed that the transmitter part was able to measure the three river water variables and sent them to the receiver part which was placed 100 metres apart. The measurement results of the field testing showed that with the relatively unchanged river condition the measurement results of the proposed systems were consistent without any abrupt change. Considering its weaknesses, the future research should address the construction, power sources, and data analytics including sending warning messages when the data is not in the correct range.

\section{ACKNOWLEDGEMENT}

Authors would like to thank to DRPM Kemenristek/BRIN Republic of Indonesia for supporting this research with the scheme of Penelitian Dasar Unggulan Perguruan Tinggi (PDUPT) year 2020.

\section{REFERENCES}

1. B. P. Resosudarmo. River water pollution in Indonesia: an input-output analysis, International 
Journal of Environment and Sustainable Development, vol. 2, no. 1,pp. 62-77, 2003.

2. M. Pule, A. Yahya, and J. Chuma. Wireless sensor networks: A survey on monitoring water quality, Journal of Applied Research and Technology, vol. 15 (2017), pp. 562-570, 2017.

3. M. N. M. Yasin, M. M. A. M. Hamzah, M. Kassim, and N. Arbain. Freshwater pH level control and GUI system for prawn breeding, International Journal of Advanced Trends in Computer Science and Engineering (IJATCSE), vol. 9, no.4, pp. 5887-5893, July-August 2020 .

4. A. M. Dunca. Water Pollution and Water Quality Assessment of Major Transboundary Rivers from Banat (Romania), Journal of Chemistry, vol. 2018, pp. $1-8,2018$

5. K. A. Mamun, F. R. Islam, R. Haque, M. G. M. Khan, A. N. Prasad, H. Haqva, R. R. Mudliar, and F. S. Mani. Smart water quality monitoring system design and KPIs analysis: case sites of Fiji surface water, Sustainability 2019, vol. 11, 7110, pp. 1-21, 2019.

6. Y. Martinus, W. Astono, D. Hendrawan. Water quality study of Sunter River in Jakarta, Indonesia, IOP Conf. Series: Earth and Environmental Science, vol. 106 (2017) 012022, pp. 1-6, 2017.

7. M. Barabde, and S. Danve. Real Time Water Quality Monitoring System, International Journal of Innovative Research in Computer and Communication Engineering, vol. 3, no. 6, pp. 5064-5069, June 2015.

8. A. T. Demetillo, M. V. Japitana and E. B. Taboada. A system for monitoring water quality in a large aquatic area using wireless sensor network technology, Sustainable Environment Research (2019), vol. 29:12, pp. 1-9, 2019.

9. O. Elijah, T. A. Rahman, C. Y. Leow, H. C. Yeen, M. A. Sarijari, A. Aris, J. Salleh, and T. H. Chua. A concept paper on smart river monitoring system for sustainability in river, International Journal of Integrated Engineering, vol. 10, no. 7, pp. 130-139, 2018.

10. V. V. Daigavane, and M. A Gaikwad. Water quality monitoring system based on IoT, Advances in Wireless and Mobile Communications, vol. 10, no. 5, pp. 1107-1116, 2017.

11. S. Pappu, P. Vudatha, A. V. Niharika, T. Karthick, and S. Sankaranarayanan. Intelligent IoT based water quality monitoring system, International Journal of Applied Engineering Research, vol. 12, no. 16, pp. 5447-5454, 2017.

12. J. Ijaradar, and S. Chatterjee. Real-time water quality monitoring system, International Research Journal of Engineering and Technology (IRJET), vol. 05, no. 03, pp. 1166-1171, Mar-2018.

13. S. Geetha, and S. Gouthami. Internet of things enabled real time water quality monitoring system, Smart Water (2017), vol. 2:1, pp. 1-19, 2017.
14. M. J. V. Kumar, and K. Samalla. Design and development of water quality monitoring system in IoT, International Journal of Recent Technology and Engineering (IJRTE), vol. 7, no. 5S3, pp. 527-533, February 2019.

15. M. Li, C. Lin, J. Ren and F. Jiang. A wireless ecological aquaculture water quality monitoring system based on LoRa technology, Advances in Computer Science Research, vol. 89, pp. 5-7, 2019.

16. M. S. U. Chowdury, T. B. Emran, S. Ghosh, A. Pathak, M. M. Alam, N. Absar, K. Andersson, and M. S. Hossain. IoT based real-time river water quality monitoring system, Procedia Computer Science, vol. 155 (2019), pp. 161-168, 2019.

17. B. Mataloto, J. C. Ferreira, R. Resende, R. Moura, and S. Luís. BIM in People2People and Things2People interactive process, Sensors 2020, vol. 20 2982, pp. 1-18, 2020.

18. H. Supriyono, E. D. Febriyanto, and K. Harismah, Portable machine to machine system for monitoring temperature and flammable gas of outdoor environment, in AIP Conference Proceedings, vol. 2114 040014 (2019). Surakarta; 2019, pp. 040014-1040014-8, 2019.

19. Supriyono, H., and Saiful Arifin Wahyu Wibowo. 2020. Battery Voltage and Room Temperature Monitoring Using Website with Warning Notification for Pagardewa Compressor Station, International Journal of Advanced Trends in Computer Science and Engineering (IJATCSE), vol. 9, no.4, pp. 5376-5383, July-August 2020.

20. M. Carminati, A. Turolla, L. Mezzera, M. Di Mauro, M. Tizzoni, G. Pani, F. Zanetto, J. Foschi and M. Antonelli. A self-powered wireless water quality sensing network enabling smart monitoring of biological and chemical stability in supply systems, Sensors 2020, vol. 20, 1125, pp. 1-16, 2020.

21. R. J. Basan, K. T. Arizobal, N. C. T. Obas, and R. S. Pangantihon. Development of toilet air quality assessment and monitoring system, International Journal of Advanced Trends in Computer Science and Engineering (IJATCSE), vol. 9 no. 4, 6821-6828, July August 2020. 